\title{
Morfologia eSTRUTURAL CAE ViA MÉtodo dos ElEMENTOS Finitos "FEM": UM ESTUDO DE CASO FOCADO NA ANALISE DE IMPACTO
}

\author{
Evandro Secchi de Oliveira ${ }^{1}$ e Roderley Camargo $^{2}$ \\ ${ }^{1}$ UNISAL Centro Universitário Salesiano de São Paulo \\ ${ }^{1}$ Bosal do Brasil Ltda \\ ${ }^{2}$ Robert Bosch Ltda
}

E-mails: evandro.oliveira@bosal.com.br, roderley.camargo@br.bosch.com

\section{RESUMO}

O Sistema de exaustão automotivo, está constantemente sofrendo solicitações, oriundas da vibração, ocasionada pelo motor e pelo chassi do veículo. Por este motivo, é imprescindível desenvolver um método matemático, que simule as condições físicas deste produto, para que seja possível prever na fase de projeto, todos as reações presentes no escapamento. Neste sentido, apresenta-se neste trabalho, um estudo de análise modal, contemplando as frequências naturais, para cada suporte de fixação do escapamento. O modelo matemático CAD (Computer Aided Design) foi desenvolvido em ambiente CREO enquanto a analise CAE (Computer Aided Engineering) foi feita no ambiente ABAQUS, e utilizando o método dos elementos finitos. A análise CAE foi desenvolvida e otimizada por intermédio dos aplicativos Hypermesh e Hyperview respectivamente. O uso deste método, possibilitou verificar a ocorrência da frequência crítica de excitação, menor que $200 \mathrm{~Hz}$. Caso ocorra, há que se retrabalhar o componente, para que o mesmo esteja fora da faixa determinada como critério de falha. Finaliza este trabalho apresentando algumas perspectivas futuras, fazendo referência ao método de correlação "hammer impact".

\section{INTRODUÇÂO}

O Sistema de exaustão utilizado nos veículos ciclo OTTO, tem por finalidade atenuar os ruídos oriundos do motor, diminuir os gases nocivos a saúde por meio de um catalisador, e arrefecer os gases antes da liberação para a atmosfera. Este conjunto é localizado na parte inferior do veículo e interligado ao motor, com isto, todo o conjunto está sujeito as vibrações ocasionados pelo movimento do motor, e pelo chassi do veículo, quando o mesmo está em movimento.

Assim, a partir da simulação das condições de contorno, definidas como representativas da vida operacional do conjunto do escapamento, e de posse do modelo matemático tridimensional CAD, utilizou-se a técnica dos elementos finitos como forma de prever o comportamento elástico deste produto. Realizou-se estudos empíricos nos veículos por parte das montadoras, em que foi determinado e documentado em normas, a frequência natural mínima aceitável para que os componentes do veículo, não entrem em um estado de ressonância ou próximo a ressonância onde ocorrerá a falha. Para este estudo, foi utilizado um sistema de exaustão de um carro compacto, cuja motorização foi de 4 cilindros 1.0 litro e o 
critério de falha utilizado foi a máxima frequência de $200 \mathrm{~Hz}$, no primeiro modo de vibração. Os componentes analisados foram os hangers (ganchos), que são responsáveis pela fixação do escapamento no chassi veicular. Neste método, a prerrogativa é obter o primeiro modo de vibração maior que $200 \mathrm{~Hz}$. Nos dados de entrada do software, foi levado em consideração a especificação do material do hanger, dos tubos onde os mesmos são soldados e as especificações das soldas.

Com o software Hypermesh, foram criadas as malhas e as condições de contorno, posteriormente, foram inseridas todas as informações dos materiais como: espessura, modulo de elasticidade, densidade e coeficiente de Poisson. Para rodar a simulação e obter os desejados modos de vibração, foram utilizados os algoritmos internos do solver Abaqus. No final, utilizou-se o software Hyperview para analisar os dados de saída do Abaqus e gerar os relatórios correlatos.

Estes relatórios serão de extrema importância, para a estratégia de projeto, por parte do engenheiro do produto, pois visto isto, o mesmo poderá verificar o projeto ainda na fase de pré-desenvolvimento do produto, conseguindo poupar tempo no que diz respeito a construção de protótipos, e testes físicos, evitando ou minimizando futuros retrabalhos de projeto [1]. Também poderá ser levado em conta a manufatura enxuta, conseguindo construir um produto com um mínimo de material, sendo reforçado apenas nas áreas que necessitam de robustez estrutural.

\section{INFORMAÇÔES GERAIS}

Para se desenvolver produtos inovadores e bem-sucedidos no atual cenário global e competitivo, há que se ter recursos de informática cientifica e fazer uso de simulação. Neste senso, para que a empresa sobreviva neste ambiente de competição, a simulação deve fazer parte do projeto, já na fase inicial do processo de desenvolvimento do produto [2] e [3]. A capacidade de se analisar as características multi-corpos do desempenho físico de um projeto, antes de criar um primeiro protótipo, pode aumentar de forma significativa a produtividade. As empresas precisam de ferramentas de simulações robustas, o suficiente para superar com eficácia, as demandas de prazo, custo e qualidade final do produto [4].

A análise integrada aos programas de $\mathrm{CAD}$, estão transformando o método dos elementos finitos em uma ferramenta onipresente, e seu uso será cada vez mais similar ao de um motor de um automóvel: todos poderão usá-lo apenas com um entendimento básico de seu funcionamento [5]. Poucos dos usuários realmente precisarão entender o método, para extrair dele um melhor desempenho e confiabilidade.

A tecnologia de simulação permite a utilização de modelagem matemática, baseada em computador, para aproximar e simular os fenômenos complexos do universo físico, tendo como dados de entrada, o modelo CAD 3D [6]. De posse do modelo matemático CAD, enviado pela montadora, inicia-se o processo de construção da malha de elementos finitos, em que é possível indicar para cada parte da geometria CAD, o tipo de elemento e tamanho adequado para cada região desta geometria. Este processo é o início da formulação CAE via método dos elementos finitos. Depois de inseridas todas estas informações, é analisado a resposta gerada pelo solver, validando ou reprovando o design atual. 
As conclusões e desdobramentos deste trabalho, estão destacados em detalhes no tópico estudo de caso.

\subsection{METODOLOGIA}

A metodologia utilizada foi a aplicação da técnica FEA (Finite Element Analize) e verificando os modos de vibração de cada componente, antes da construção do protótipo. O espectro da análise e síntese do modelo matemático 3D, foi obtido utilizando softwares próprios para geração de malhas, relatórios e cálculos. O Hypermesh foi utilizado para a geração da malha, que utiliza como base, a geometria gerada em formato neutro de dados ".stp". Desta forma, permite inserir toda a informação de material e condição de contorno, no padrão em que o software ABAQUS utiliza para o cálculo. Então, três etapas complementam este método: Input, Solver e Output [7].

Na fase de Input, são considerados os seguintes requisitos: Geometria, Material, Restrições, Propriedades físicas e Elementos.

Na fase de Solver, o software utiliza um algoritmo interno para a convergência dos cálculos, conforme a quantidade de iterações mínima e máxima atribuídas.

$\mathrm{Na}$ fase de Output, será considerado como resultado, o deslocamento e os modos de vibrações. Neste momento, a intenção é convalidar estes resultados conforme o requisito da montadora, especificamente mantendo o primeiro modo de vibração do componente, acima da faixa de $200 \mathrm{~Hz}$.

A Figura 1 ilustra as macros etapas da análise FEA: Input, Solver e Output.

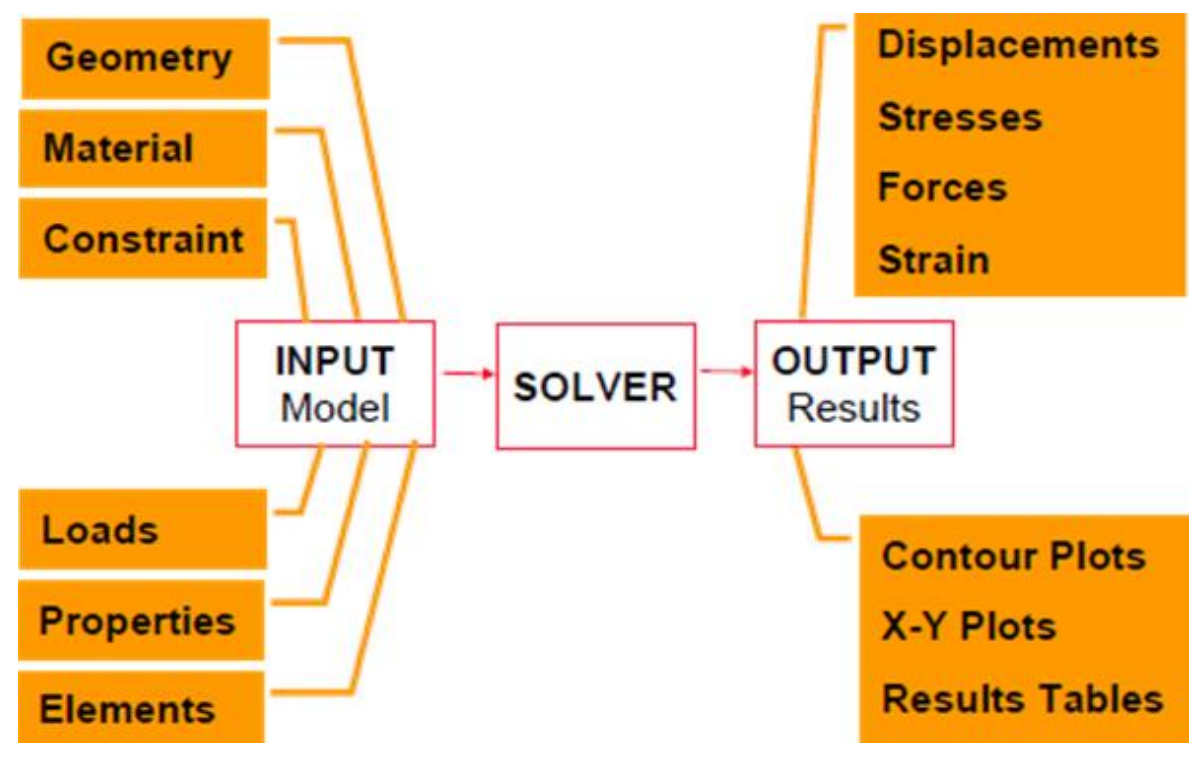

Figura 1 - Macro etapas da análise FEA [7]

Quanto ao delineamento, a metodologia assume uma abordagem orientada ao estudo de caso, em que será demonstrado via projeto de simulação virtual, e refazendo o projeto conforme o output da simulação virtual. 


\subsection{FUNDAMENTAÇÂO TEÓRICA}

A vibração é um fenômeno estudado pelas civilizações a mais de 3000a.c. anos. No passado, a humanidade se preocupava com este fenômeno apenas para a construção de instrumentos musicais. Porém, com o passar do tempo, estudos foram mostrando que o fenômeno de vibração, sempre esteve presente na vida dos seres humanos.

Cita-se como exemplo, a capacidade que temos de poder sentir as ondas sonoras, e ao mesmo tempo, ouvir estas mesmas ondas vibrando no tímpano. A respiração está associada com a vibração dos pulmões e o andar envolve o movimento oscilatório das pernas e das mãos.

Porém, o fenômeno de vibrações na engenharia, está sempre associado com o desconforto gerado por ruídos, ou perda de eficiência de uma máquina, ocasionada por desbalanceamento ou desgastes de componentes [8]. Também vale a pena ressaltar, o problema de engenharia causado por ressonância, onde uma vibração externa pode entrar na mesma frequência que a máquina ou estrutura, ocasionando o colapso da mesma.

Os primeiros esforços dos cientistas para resolverem os problemas de vibrações, foram o de criar um modelo matemático, que descrevesse as vibrações físicas e a determinação das frequências naturais. Um dos métodos mais comuns utilizados para a determinação das frequências naturais é o método de Jacobi, que é um método iterativo e produz todos os autovalores e auto vetores simultaneamente [8].

Este método é baseado em um teorema da álgebra linear, que afirma que uma matriz simétrica real [D], tem somente autovalores reais, e que existe uma matriz ortogonal real [R] tal que $[R]^{T}[D][R\}$ seja diagonal. Os elementos da diagonal são os autovalores, e as colunas da matriz [R] são os auto vetores. De acordo com o método de Jacobi, a matriz [R] é gerada como um produto de várias matrizes de rotação, da forma onde todos os elementos, exceto os que aparecem nas colunas e linhas i e j, são idênticos aos da matriz identidade [I]. Se as entradas de seno e co-seno aparecerem nas posições (i,i), (i,j), (j,i), e (j,j), então, os elementos correspondentes de $\left[R_{1}\right]^{T}[D]\left[R_{1}\right]$ podem ser calculados da seguinte maneira:

$$
d_{i j}=d_{i i} \operatorname{sen}^{2} \theta-d_{i j} \operatorname{sen} \theta \cos \theta+d_{j j} \cos ^{2} \theta
$$

Se escolhemos " $\theta$ " como:

$$
\operatorname{tg} 2 \theta=\left(\frac{2 d_{i j}}{d_{i i}-d_{i j}}\right) d_{i j}
$$

Então, resultará que:

$$
d_{i j}=d_{j i}=0
$$

Assim, cada etapa do elemento de Jacobi, reduz um par de elementos fora da diagonal, a zero.

O método dos elementos finitos consiste em discretizar a geometria que se deseja estudar, criando elementos cujas dimensões e formatos são conhecidos por todo o volume ocupado por 
esta geometria [9]. Estes elementos estarão interligados entre si, por meio de nós e referenciados por um sistema de coordenada global. Cada nó é identificado e é inserido em uma matriz, por meio da sua coordenada. Por isto, quanto menor for o elemento, serão necessários mais elementos para preencher todo o volume da geometria em estudo, e por consequência, serão criados mais nós aumentando também o tamanho da matriz.

Com isto, é possível aumentar a precisão do resultado obtido, até chegar a um determinado número de elementos, que não influenciará no resultado. Este procedimento tem o inconveniente de ocupar muito tempo de cálculo do computador. Então, é preciso levar em conta o tamanho do elemento ideal, para obter um resultado coerente, assim como, obter o menor tempo de cálculo possível, em função do número de nós.

O software irá interpretar cada um destes nós e criar uma outra matriz de rigidez, com as informações do material, para inserir a rigidez em cada nó, criando assim, a matriz de propriedades da geometria. Com estas informações, o solver começa a criar uma "perturbação inicial" na geometria em estudo, até que a mesma comece a deslocar os nós a uma determinada frequência, criando o primeiro modo de vibração. O software continua a perturbar os nós da geometria, aumentando a frequência até que os nós comecem a se movimentar de uma outra forma, indicando o segundo modo de vibração. O software vai aumentando a frequência até atingir todos os modos de vibração indicados previamente para a análise modal [10].

\section{ARQUITETURA BÁSICA DO MÉTODO DOS ELEMENTOS FINITOS}

A confiabilidade dos resultados, obtidos a partir do uso da metodologia FEA, se comparada aos diversos outros métodos empregados nos dias de hoje, se resume na facilidade de interface com o software de analise. O programa de elementos finitos busca na simplificação de operação, uma maneira de adequar os recursos de equações matemáticas e orientá-las ao objeto de analise [11].

Assim, três etapas contemplam uma programação via FEA, como forma de orientação a lógica computacional, quais sejam [11]:

\section{INPUT $\rightarrow$ PREPROCESSING $\rightarrow$ SOLVER}

A fase de Input contempla os dados do material e os requisitos do projeto, quais sejam: Geometria, Material, Forças, Restrições e Tipos de elementos. Nesta fase, é de suma importância a representação gráfica da geometria do modelo CAD, com os principais detalhes construtivos, envolvendo a fabricação, montagem e o projeto de fato. Também é importante a entrada dos dados do material a ser utilizado na análise. Faz parte deste tópico: Módulo de elasticidade, Coeficiente de Poisson e Densidade do material. Além disso, a leitura das condições de contorno, podem ser feitas nesta fase, assim como as condições de carregamento também são introduzidas nesta etapa.

A fase do PREPROCESSING diz respeito a preparação da malha e dos elementos, necessários para discretizar o modelo matemático e iniciar os cálculos. Nesta fase, também é organizado toda as propriedades dos componentes, indicando se este foi construído originalmente em forma de chapa, solido maciço ou sólido de geometria variada. Também é indicado os 
parâmetros em que o solver irá trabalhar, como por exemplo, a quantidade de modos de vibração máxima a serem encontradas, quantidade de incrementos, acuracidade de calculo e tolerância interna de banda.

$\mathrm{Na}$ fase de solver, é utilizado o algoritmo interno do ABAQUS para a solução do sistema, e tem-se então, o resultado da análise estrutural em diversos formatos de saída, de acordo com o critério de falha, determinado no escopo da analise. São exemplos de saída de resultados: Tensões, Deformações, Deslocamentos, Gráficos, Diagramas, Temperaturas, Pressões entre outros.

A Figura 2 ilustra o fluxograma da arquitetura do método FEA para este caso de estudo.

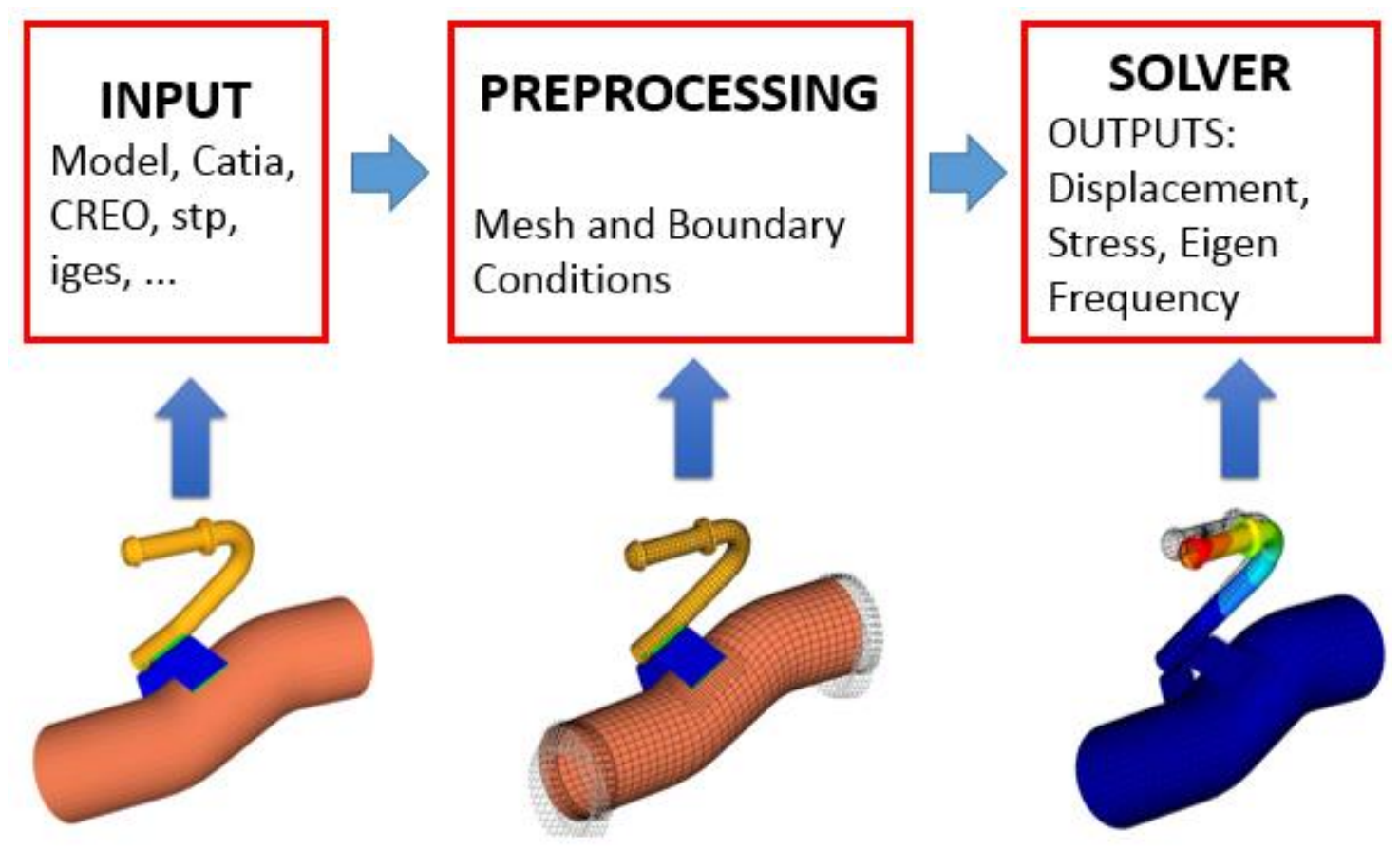

Figura 2 - Arquitetura computacional do método FEA [11]

A saída gráfica dos resultados, incluindo os aspectos de simulação, dão ao engenheiro analista, a noção exata do que está ocorrendo em termos de realidade aumentada, e convergindo para uma solução computacional robusta, no que se refere as condições de entrada [12].

\subsection{DEFINIÇÃO DA TÉCNICA DOS ELEMENTOS FINITOS}

É uma técnica matemática que consiste em discretizar uma estrutura, - modelo matemático tridimensional - em elementos paramétricos ou isoparamétricos conhecidos e conectados por "nós". As propriedades dos Materiais e Elementos são especificadas a fim de representarem as propriedades físicas do modelo.

A Figura 6 ilustra a concepção da técnica FEA, evidenciando os Nós, os Elementos e a Estrutura, que será encapsulada ao método [6]. 


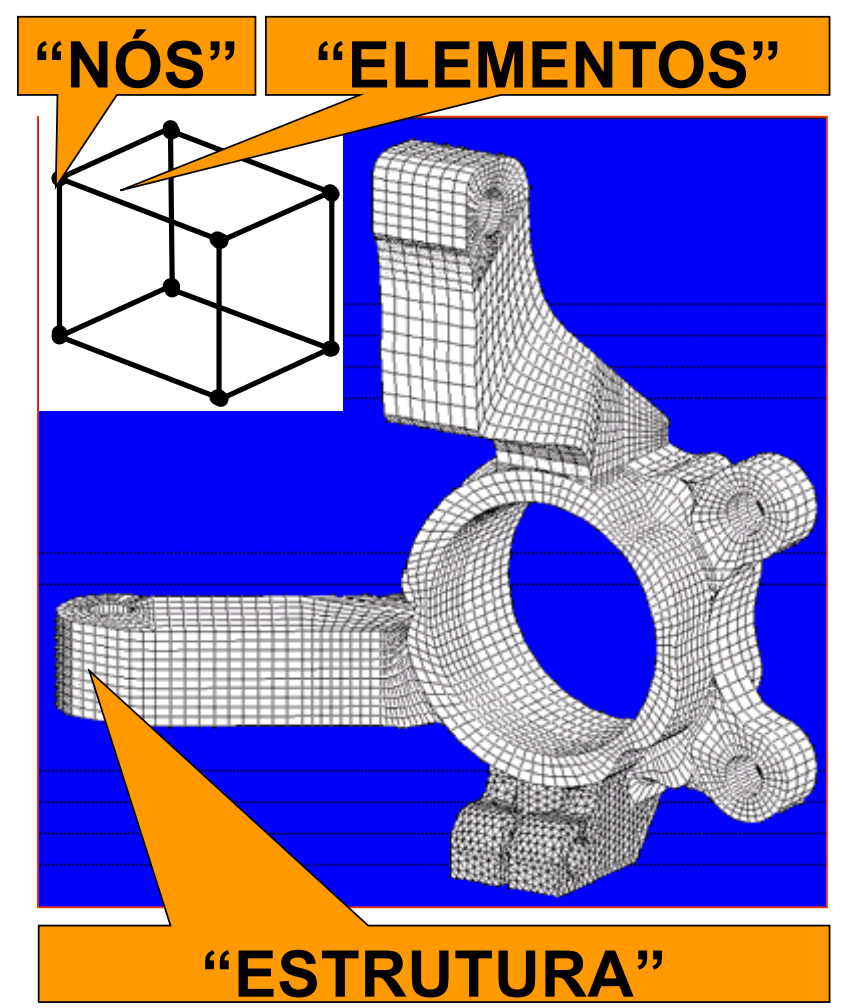

Figura 3 - Definição de estrutura, elementos e "nós" [5]

Também há que se envolver todo o modelo matemático, inserindo as mesmas condições de contorno, consideradas no protótipo, e refinando a malha nos locais em que haja relevância, para que o resultado desta análise, venha a convergir com o resultado do teste.

Ao inserir as condições de contorno, os nós gerados inicialmente sofrerão uma excitação, ou seja, um deslocamento entre os próprios nós. O solver irá interpretar estes deslocamentos, tratando cada nó como um vetor, e a partir daí, cria-se uma matriz que por sua vez, indica o sentido de deslocamento em cada nó e a frequência em que o mesmo começou a se deslocar.

\subsection{PASSOS PARA A ANÁLISE FEA}

A analise FEA pressupõe algumas etapas para a correta aplicação das condições de contorno de acordo com Figura 3, quais sejam: Modelamento geométrico, Preparação das malhas, Definição da simulação, Solver, Avaliação dos resultados e Otimização [6].

O modelamento geométrico compreende a vetorização em ambiente CAD da peça que se deseja submeter a análise, sendo comumente denominada de modelo 3D.

A fase de preparação da malha e condições de contorno, equivale a discretização da estrutura em elementos paramétricos ou isoparamétricos, e conhecidos as suas características matemáticas. Também é nesta fase em que se determina, os diversos tipos de carregamentos que a estrutura irá submeter, assim como as suas restrições, dando origem aos graus de liberdade.

Para a definição da simulação, é preciso especificar que tipo de trabalho computacional será realizado, podendo ser: Estática, Dinâmica, Modal, Térmica, CFD, RSA, entre outras. 
O próximo passo é o solver, que é a etapa de trabalho computacional, envolvendo todo o algoritmo matemático das diversas especialidades da engenharia, com a intenção de validar os limites por exemplo da lei de Hooke, Young, Poisson, entre outros cientistas.

Segue com a avaliação dos resultados, em que o sistema após ter realizado os cálculos internos, devolve as informações com os resultados de vibração, deslocamento, tensão, temperatura, pressão, deformação, etc.

Finaliza esta etapa com uma argumentação: Validar ou Otimizar o projeto? Em que o engenheiro calculista define o que será feito, com base no resultado da simulação computacional e analise numérica dos dados.

A Figura 4 denota a topologia computacional da metodologia FEA, utilizada neste trabalho.

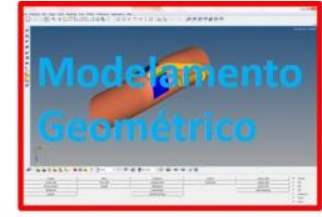

Modelo 3D

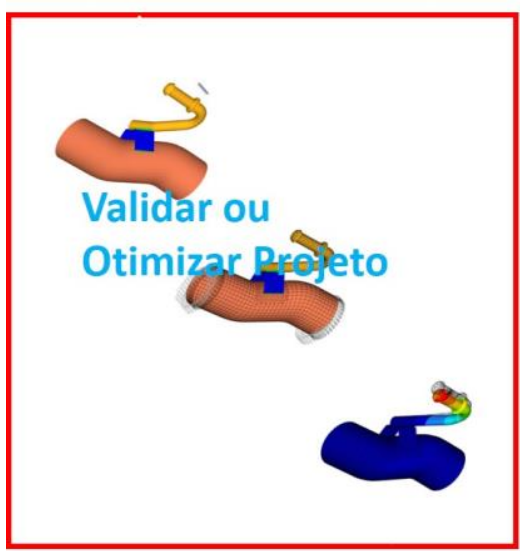

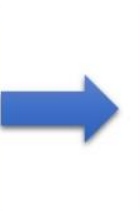

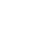

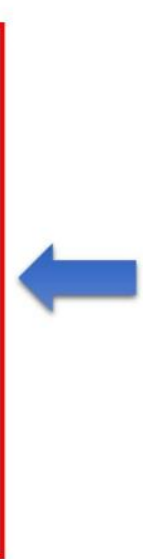

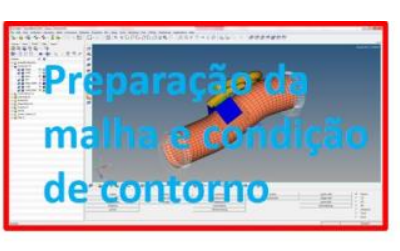

Quad, tetra ou Trias

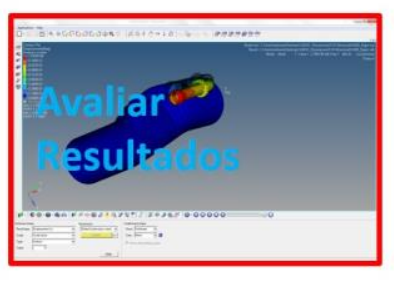

Modo de vibração,

Deslocamento, Tensão,

Temperatura, etc

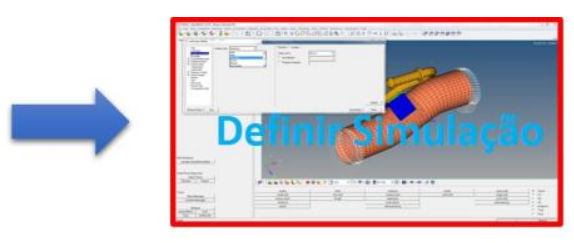

Estática, Dinâmica, frequência, Térmica, CFD

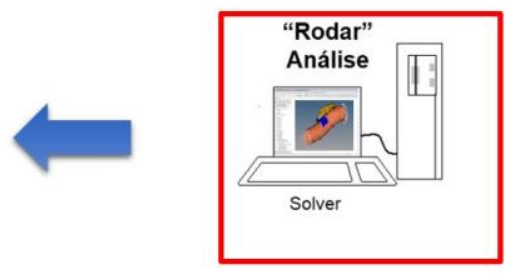

Figura 4 - Topologia computacional da analise FEA

\section{ESTUDO DE CASO: ANÁLISE MODAL DOS HANGERS}

A formulação das malhas foram geradas a partir do modelo CAD 3D do conjunto, em que descartou-se as regiões de não interesse, deixando apenas as malhas de cada Hanger em arquivos ".inp" ou seja, arquivos de inputs para o ABAQUS, os quais contem os elementos de malha, condições de contorno e informações de materiais.

No total, foram gerados 4 arquivos para cada um dos 4 Hangers que compõem o sistema. Trabalhou-se com um elemento do tipo "QUAD" com tamanho médio de 5x5, deixando como parâmetro aceitável, apenas 5\% de elementos "triangulares". Estes elementos triangulares foram evitados, pelo fato de que pela configuração da sua geometria, favorece a concentração de grandes tensões.

Para as propriedades dos materiais de chapas, utilizou-se a propriedade de "SHELL" com a espessura de cada material.

Para as soldas, foram utilizadas as propriedades de "SOLIDS", pelo fato de serem usados elementos do tipo "QUAD 3D”, cuja finalidade principal é a interligação dos componentes. 
A Figura 5 ilustra as quatro malhas geradas para o componente hanger.
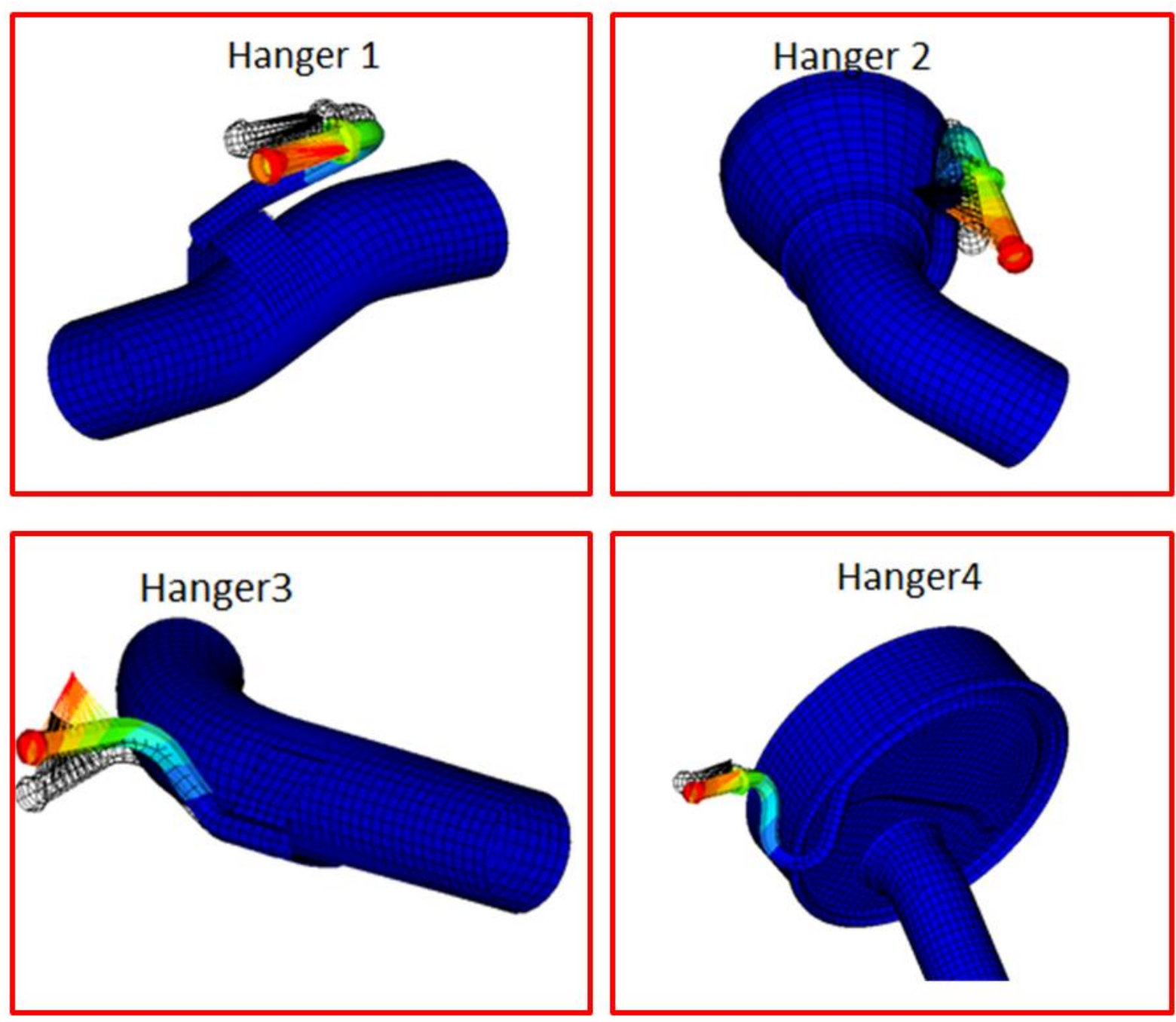

Figura 5 - Arquivo com as Malhas geradas

Foram considerados também para a simulação, as especificações dos "rubbers" ou seja, os coxins que interligam os ganchos com o chassi, e que também atuam como um amortecedor para o sistema de exaustão. Devido ao fato que esta se considerando para este caso, os modos de vibração dos hangers, com o intuito de se analisar qual a mínima frequência, para que estes componentes comecem a vibrar, foi inserido apenas a massa do coxim em um elemento "1D", interligado por elementos "1D Rígidos", em todos os nós do local onde o coxim está instalado.

Neste caso, a massa inserida foi de $4 \times 10^{-6}$ toneladas, conforme o sistema de unidades trabalhadas para este conjunto. Lembrando que o software trabalha de forma adimensional, ou seja, o usuário é responsável por indicar as unidades e escalas adequadas, que se deseja trabalhar.

A Figura 6 denota a representação gráfica do elemento do tipo "1D”, distribuindo a massa do coxim entre os nós adjacentes que formam o conjunto do hanger. 


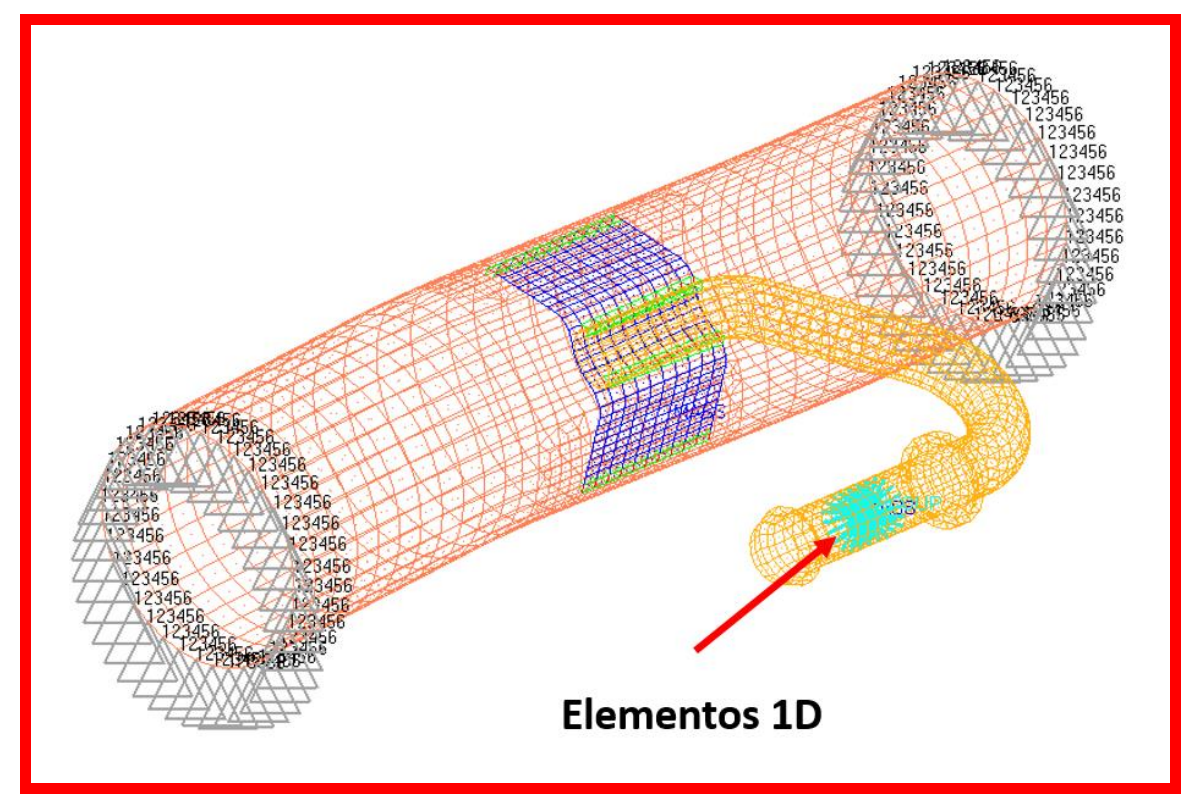

Figura 6 - Elementos 1D, distribuindo a massa do coxim entre os nós

Definida a condição de contorno mais representativa para o modelo matemático, o próximo passo foi configurar o solver para a condição de Frequency, com no mínimo 5 modos de vibração.

Com o solver devidamente configurado, iniciou-se a análise computacional, em que o solver compilou os cálculos da análise modal no ambiente ABAQUS. Após a finalização dos cálculos, o solver retornou e apresentou os resultados graficamente, indicando no modelo matemático $\mathrm{CAD}$, os deslocamentos $(\Delta \mathrm{l})$ em cada modo de vibração, assim como a frequência natural $(\mathrm{Hz})$ correlata.

A Figura 7 ilustra o deslocamento do hanger ampliado em uma escala de 2 vezes.

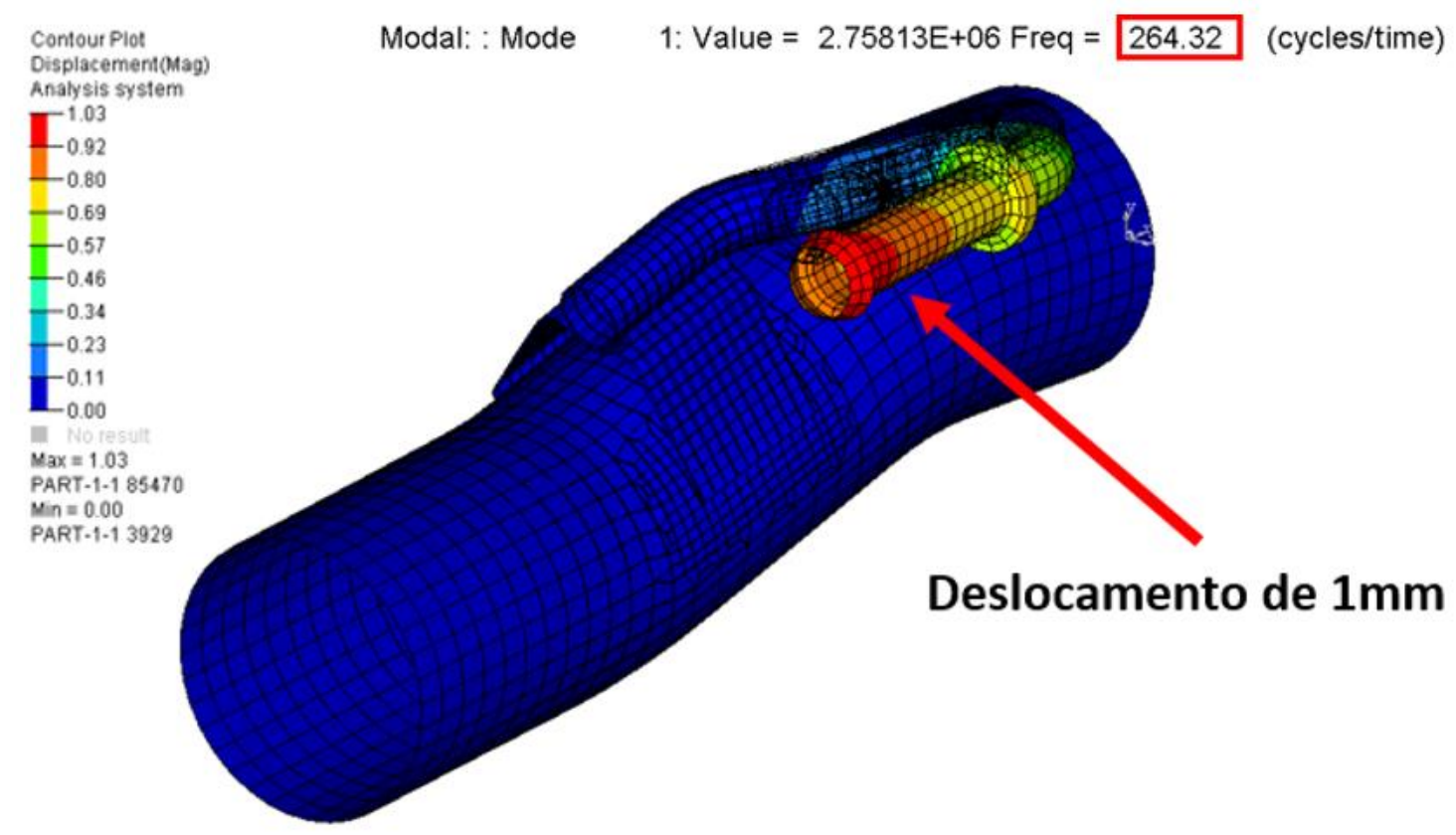

Figura 7 - Deslocamentos $(\Delta \mathrm{l})$ e modos de vibrações $(\mathrm{Hz})$ 
A Figura 8 ilustra os modos de vibrações encontrados em cada hanger, evidenciando a localização geométrica e o valor da frequência encontrada em Hertz.
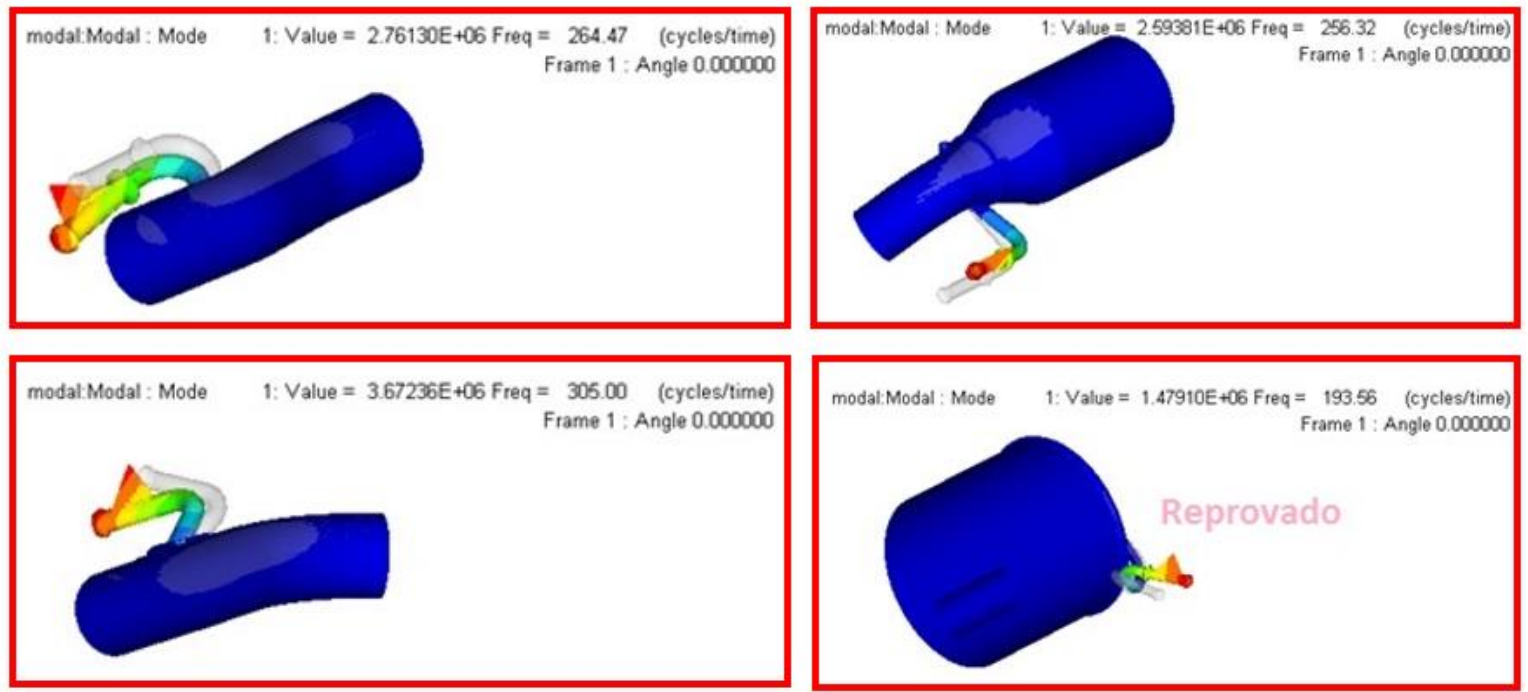

Figura 8 - Modos de vibrações em cada hanger

No final da análise, foi possível verificar que o hanger 4 estava com uma frequência natural abaixo de $200 \mathrm{~Hz}$, o que compromete seriamente o conjunto do escapamento. Assim, é necessário criar um retrabalho, ou melhor dizendo, uma otimização geométrica do componente, para adequar este hanger a uma faixa de frequência ideal.

Visto isto, foi inserido mais um cordão de solda na parte oposta do atual cordão, e que prende este hanger no baffle do silenciador traseiro. Este cordão de solda irá aumentar a massa do sistema, fazendo com que o mesmo aumente sua frequência natural de vibração.

A Figura 9 denota a comparação entre a modificação do componente e o componente atual.
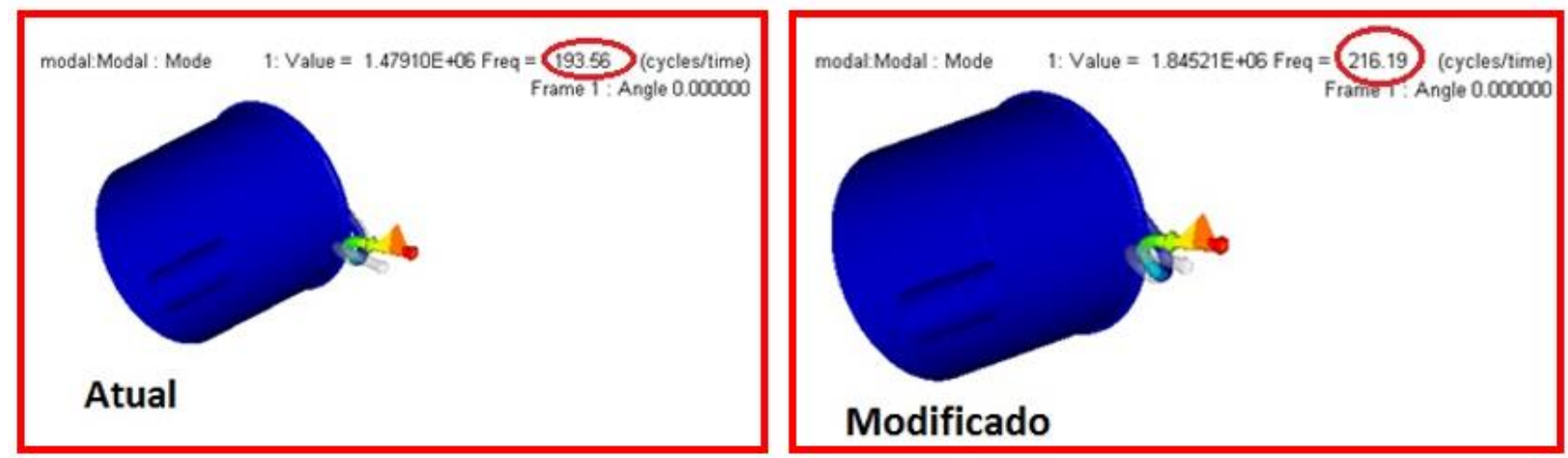

Figura 9 - Protótipo confeccionado para o teste

Com a finalização das análises, foi possível verificar se os componentes atendem a especificação do cliente, ainda em plena fase de Pré-Desenvolvimento. Nesta fase, as cotações para a definição do custo final do produto ainda não foram congeladas, e desta forma, com as modificações feitas de forma virtual, o projeto poderá ser conduzido com uma maior garantia 
de confiabilidade de função. Também espera-se uma decisão sem a dúvida de que será necessário acrescentar material ou adicionar mais algum componente, onerando o preço do produto. Por outro lado, o protótipo será criado, sem que seja necessário retrabalhos, contribuindo desta maneira, para a redução do custo do projeto.

\subsection{BULK DATA}

Para esta analise, utilizou-se um processador Intel Xeon W3565, 3.2 GHz, 64 bits, $10 \mathrm{~GB}$ RAM e disco rígido de 3,5 TB e 4 GB de swap_space, além de:

$\begin{array}{ll}\text { - Tempo de processamento total } & =13 \mathrm{~min} \\ \text { - No. de Elementos } & =2946 \\ \text { - No. de Nós } & =2910 \\ \text { - No. de Variáveis } & =17460\end{array}$

\section{CONCLUSÃO}

O resultado da simulação, indicou qual componente seria necessário uma melhoria para adequar o produto as especificações do cliente, resultando em uma maior clareza para a cotação do valor final do mesmo, e diminuindo o tempo de reprojetos e validações, com construções de protótipos. Com este método, foi possível comprovar para a montadora, que o produto foi concebido a partir de métodos robustos, e atende as especificações, diminuindo a incerteza de problemas futuros, durante as fases do processo de desenvolvimento do produto.

Futuramente, poderá ser utilizado um método de correlação denominado "Hammer Impact", que constitui um "martelo de impacto", instrumentado com um sistema de medição LMS, em que, após uma pequena perturbação no componente, é possível verificar a frequência natural do mesmo, e correlacionar com a solução encontrada na simulação computacional.

\section{REFERÊNCIAS}

[1] Back, N., Ogliari, A., Dias, A., Da Silva, J.C.: Projeto Integrado de Produtos: Planejamento, Concepção e Modelagem, ISBN: 9788520422083, Editora Manole, 2008

[2] Rozenfeld, H., Forcellini, F.A., Amaral, D.C., Toledo, J.C., Silva, S.L., Alliprandini, D.H., Scalice, R.K.: Gestão de Desenvolvimento de Produtos: Uma referência para a Melhoria do Processo, Saraiva, São Paulo, 2006

[3] Pahl, G., Beitz, W., Feldhusen, J., Grote, K.-H.: Projeto na Engenharia, $1^{\text {a }}$ ed., Edgard Blücher, São Paulo, 2005

[4] Ehrlenspiel, K., Kiewert, A., Lindemann, U.: Cost-Efficient Design, ASME Press, ISBN 0-7918-0250-7, New York, 2007

[5] MacNeal, R.: MSC/NASTRAN for Window: Finite Element Modeling, MacNeal Schwendler Corporation, San Diego/Califórnia, 1997

[6] Camargo, R.: Topologia de Projeto Robusto aplicando o método dos Elementos Finitos, Revista Ciência e Tecnologia, v. VII, p. 13-29, 2004 
[7] Alves Filho, A.: Elementos Finitos - A Base da Tecnologia CAE, Editora Erica, $1^{\circ}$ Edição, São Paulo, 2000

[8] Rao, S.: Vibrações Mecânicas, Quarta Edição, Pearson, 2009

[9] Zienkiewicz, O. C. e Taylor, R. L.: Finite Element Method- Basic Formulation and Linear Problems, Vol.1, McGraw-Hill Co., New York, 1989

[10] Thomson, W. T.: Teoria da Vibração com Aplicação, Rio de Janeiro, Interciencia, 1978

[11] Hughes, T. J. R.: Finite Element Method - Linear Static and Dynamic Finite Element Analysis, Prentice-Hall, Englewood Cliffs, 1987

[12] Fonseca, J.S.O, Selk, C.A.C., Pereira, L.T.V., Marczak, R.J., Alves, D.B., Luersen, M.A.: Ferramentas de Simulação em Mecânica: Elementos Finitos, UFRGS, 2002 